\title{
Calculation Scalar Curvature of the Cycloid Surface in
} $\mathfrak{R}^{5}$

\author{
Wageeda Mohamed Mahmoud*, Samar Moukhtar and Yassien Gulab Gouda \\ Deptartment of Mathematics, Faculty of Science, Aswan University, Egypt
}

\begin{abstract}
In this work, we show a local study of a cyclic surface in $\mathfrak{R}^{\mathbf{5}}$ produced by equiform motions of a cycloid. We definepartially such surfaces with some conjecture on its curvature. In general, we shall consider the condition that scalar curvature is locally constant. We will conclude cases of scalar curvature of following equations accounts and will put theories to explain the results of research and example.
\end{abstract}

Key words: Cycloid surface, equiform motion, scalar curvature.

\section{Introduction}

A cycloid is a curve traced by a point on the rim of a circular wheel as the wheel rolls along a straight line without slippage. It is an example of a roulette, a curve generated by a curve rolling on another curve.

The cycloid is the solution to the brachistochrone problem that it is the curve of fastest descent under constant gravity and the related tautochrone problem which the period of an object in descent without friction inside this curve does not depend on the object's starting position See [1].

An equiform transmutation in the $n$-dimensional Euclidean space $\mathfrak{R}^{\boldsymbol{n}}$ is an affine transmutation whose linear part is composed by an orthogonal transmutation and a homothetical transformation See [2-4]. Such an equiform transmutation maps points in $\chi \in \mathfrak{R}^{n}$ according to the rule:

$$
\chi \rightarrow s A x+d, A \in S(n), s \in \mathcal{R}^{+}, d \in \mathcal{R}^{n}
$$

The number $s$ is called the scaling factor. An equiform motion is determined if the parameters of Eq. (1), including $s$, are given as functions of a time parameter $t$. Then an unruffled one-parameter equiform motion moves a point $x$ via $\mathrm{X}(\mathrm{t})=\mathrm{s}(\mathrm{t}) \mathrm{A}(\mathrm{t})$ $\mathrm{x}(\mathrm{t})+\mathrm{d}(\mathrm{t})$. The kinematics corresponding to this

\footnotetext{
*Corresponding author: Wageeda Mohamed Mahmoud, Dr., research field: mathematics.
}

transformation group is called similarity kinematics [5-7].

In this paper, we study the scalar curvature of three-dimensional surfaces foliated by an equiform motion of a cycloid curve $\mathrm{m}_{0}$.

Beneath a one-parameter equiform motion of moving space $\Sigma^{0}$ with preservation a fixed space $\Sigma$. Suppose that $m_{0} \subset \Sigma^{0}$, which is moved according to an equiform motion. The point paths of the cycloid surface produce a 3-dimensional surface $\chi$, comprising the position of the starting cycloid curves $m_{0}$. At any moment, the measly transmutation of the motion will map the points of the cycloid $m_{0}$ within the velocity vectors, its end points will constitute an affine image of $m_{0}$ that transfer a subspace $\omega$ of $\mathfrak{R}^{\boldsymbol{n}}$, with $\operatorname{dim}(\omega) \leq 5$. This if the reason why we restrict our considerations to dimension $n=5$.

Let $\chi(\phi)$ be a parametrization of $m_{0}$ and $\chi(t, \phi)$ the definitive 3 -surfaces foliated by the equiform motion [8]. If we take our study of the characteristics of the motion for the utmost case $\rightarrow 0$. A prime option is when approximating $\chi(t, \phi)$ by the first derivative of the trajectories. The objective of this part is to delimit the kinematic surfaces take out with the equiform motion of a cycloid and that scalar curvature $\mathrm{K}$ is constant. The confirmation of our inference contains a precise calculation of the scalar curvature 
$\mathrm{K}$ of the surface $\chi(t, \phi)$. In this discussion, equation $\mathrm{K}=$ constant.

Furthermore, in this case, $\mathrm{K}=0$, we present the simulation of the motion of such 3-surface presenting the equations that represent the kinematic geometry. We shall discuss an example of such surface.

\section{Impersonation of the Motion}

In two copies $\Sigma^{0}, \Sigma$, of Euclidean 5 -space $\mathfrak{R}^{\mathbf{5}}$, we show a cycloid $m_{0}$ of $\Sigma^{0}$ function point at the origin that explained by:

$$
\chi(\phi)=(a(\phi-\sin \phi), a(1-\cos \phi), 0,0,0)^{T}, \phi \in \mathcal{R}
$$

Beneath a one-parameter equiform motion of $m_{0}$ in the moving space, $\Sigma^{0}$ with thought to fixed space
$\Sigma$, the position of a point $\chi(\phi) \in \Sigma^{0}$ at time $\mathrm{t}$ can be embodied in the steadfast system as:

$\chi(t, \phi)=s(t) A(t) \chi(\phi)+d(t), t \in I \subset \mathcal{R}, \phi \in \mathcal{R}(2)$ where, $\quad d(t)=\left(b_{1}(t), b_{2}(t), b_{3}(t), b_{4}(t), b_{5}(t)\right)^{\mathrm{T}}$ is the translational component of the motion at the time $\mathrm{t}, \quad A(t)=\left(a_{i j}(t)\right), 1 \leq i, j \leq 5 \quad$ is $\quad$ an orthogonal matrix and $s(t)$ executes the scaling factor of the moving system. For varying $\mathrm{t}$ and fixed $\chi(\phi), \chi(t, \phi)$ supplies a parametric description of the trajectory of $(\phi)$. Further, we believe that all concerned functions are at lowest of class $C^{1}$. From Taylor's stretching up to the first order, the adjective of the motion is giving by:

$$
\chi(t, \phi)=\{s(0) A(0)+[\dot{s}(0) A(0)+s(0) \dot{A}(0)] t\} \chi(\phi)+d(0)+t \dot{d}(0)
$$

where, "“" denotes the differentiation with regard to $t$.

As an equiform motion has an invariant point, we can suppose that the moving frame $\Sigma^{0}$ and the steady frame $\Sigma$ correspond at the zero position $\mathrm{t}=0$. Then we have $A(0)=I, s(0)=1$ and $d(0)=0$.

Hence

$$
\begin{aligned}
& \chi(t, \phi)=\left[I+\left(s^{\prime} I+\Omega\right) t\right] \chi(\phi)+t d^{\prime} \\
& \left(\begin{array}{l}
\chi_{1} \\
\chi_{2} \\
\chi_{3} \\
\chi_{4} \\
\chi_{5}
\end{array}\right)(t, \phi)=\left(\begin{array}{ccccc}
1+s^{\prime} t & t \omega_{1} & t \omega_{2} & t \omega_{3} & t \omega_{4} \\
-t \omega_{1} & 1+s^{\prime} t & t \omega_{5} & t \omega_{6} & t \omega_{7} \\
-t \omega_{2} & -t \omega_{5} & 1+s^{\prime} t & t \omega_{8} & t \omega_{9} \\
-t \omega_{3} & -t \omega_{6} & -t \omega_{8} & 1+s^{\prime} t & t \omega_{10} \\
-t \omega_{4} & -t \omega_{7} & -t \omega_{9}-t \omega_{10} & 1+s^{\prime} t
\end{array}\right)\left(\begin{array}{c}
a(\phi-\sin \phi) \\
a(1-\cos \phi) \\
0 \\
0 \\
0
\end{array}\right)+t\left(\begin{array}{l}
b_{1}^{\prime} \\
b_{2}^{\prime} \\
b_{3}^{\prime} \\
b_{4}^{\prime} \\
b_{5}^{\prime}
\end{array}\right)
\end{aligned}
$$

or in the equivalent form

$$
\left(\begin{array}{l}
\chi_{1} \\
\chi_{2} \\
\chi_{3} \\
\chi_{4} \\
\chi_{5}
\end{array}\right)(t, \phi)=a(\phi-\sin \phi)\left(\begin{array}{c}
1+s^{\prime} t \\
-t \omega_{1} \\
-t \omega_{2} \\
-t \omega_{3} \\
-t \omega_{4}
\end{array}\right)+a(1-\cos \phi)\left(\begin{array}{c}
-t \omega_{1} \\
1+s^{\prime} t \\
-t \omega_{3} \\
-t \omega_{4} \\
-t \omega_{5}
\end{array}\right)+t\left(\begin{array}{l}
b_{1}^{\prime} \\
b_{2}^{\prime} \\
b_{3}^{\prime} \\
b_{4}^{\prime} \\
b_{5}^{\prime}
\end{array}\right)
$$

For any stationary $\mathrm{t}$ in the up Eq. (4), we usually obtain an elliptic for intersection at the point $t\left(b_{1}^{\prime}, b_{2}^{\prime}, b_{3}^{\prime}, b_{4}^{\prime}, b_{5}^{\prime}\right)$. The other elliptic transitions to a two-dimensional at the cycloid surface ofform an orthogonal basis by this conditions:

$$
\omega_{2} \omega_{5}+\omega_{3} \omega_{6}+\omega_{4} \omega_{7}=0
$$

\section{Method of Curvature Scalar Calculation at the Cycloid Surfaces}

In this portion, we reckon formula of the scalar curvature of a kinematic surface in $\mathfrak{R}^{\boldsymbol{n}}$ generated by equiform motions of a cycloid satisfying the Eq. (4). The tangents vectors to the parametric curve of 
$t=$ const.,$\phi=$ const. At a kinematic surface, $\chi(t, \phi)$ is described by:

$$
\chi_{t}(t, \phi)=\left(s^{\prime} \mathrm{I}+\Omega\right) \chi(\phi)+d^{\prime}, \chi_{\phi}(t, \phi)=\left[\mathrm{I}+\left(s^{\prime} \mathrm{I}+\Omega\right) t\right] \chi^{\prime}(\phi)
$$

The first fundamental method delimited by, see [9]

Beneath the Eq. (4) we get:

$$
g_{11}=\chi_{t} \chi_{t}^{\mathrm{T}}, g_{12}=\chi_{\phi} \chi_{t}^{\mathrm{T}}, g_{22}=\chi_{\phi} \chi_{\phi}^{\mathrm{T}}
$$

$$
\begin{gathered}
g_{11}=\alpha+2 a \beta(\phi-\sin \phi)+a^{2} \gamma \phi(\phi-\sin \phi)+a^{2} \mu(\cos 2 \phi-1)+2 a(\eta+a \delta)(1-\cos \phi) \\
g_{12}=a\left(b_{1}^{\prime}+\beta t+2 a \omega_{1}+a \phi\left(s^{\prime}+\gamma t\right)\right)(1-\cos \phi)+a\left(b_{1}^{\prime}+\eta t-a \omega_{1} \phi\right) \sin \phi+a^{2} \mu t(2 \sin \phi-\sin 2 \phi) \\
g_{22}=2 a^{2}\left(1+2 s^{\prime} t+\gamma t^{2}\right)(1-\cos \phi)+a^{2} \mu t^{2}(1-\cos 2 \phi)
\end{gathered}
$$

and

$$
\begin{gathered}
\alpha=b_{1}^{\prime 2}+b_{2}^{\prime 2}+b_{3}^{\prime 2}+b_{4}^{\prime 2}+b_{5}^{\prime 2} \\
\beta=s^{\prime} b_{1}^{\prime}-\omega_{1} b_{2}^{\prime}-\omega_{2} b_{3}^{\prime}-\omega_{3} b_{4}^{\prime}-\omega_{4} b_{5}^{\prime} \\
\eta=s^{\prime} b_{2}^{\prime}+\omega_{1} b_{1}^{\prime}-\omega_{5} b_{3}^{\prime}-\omega_{6} b_{4}^{\prime}-\omega_{7} b_{5}^{\prime} \\
\gamma=s^{\prime 2}+\omega_{1}^{\prime 2}+\omega_{2}^{\prime 2}+\omega_{3}^{\prime 2}+\omega_{4}^{\prime 2} \\
\delta=s^{\prime 2}+\omega_{1}^{\prime 2}+\omega_{5}^{\prime 2}+\omega_{6}^{\prime 2}+\omega_{7}^{\prime 2} \\
\mu=\frac{1}{2}\left(\omega_{5}^{\prime 2}+\omega_{6}^{\prime 2}+\omega_{7}^{\prime 2}-\left(\omega_{2}^{\prime 2}+\omega_{3}^{\prime 2}+\omega_{4}^{\prime 2}\right)\right)
\end{gathered}
$$

The Christoffel symbols of the second kind are determined by

$$
\Gamma_{i j}^{k}=\frac{1}{2} \sum_{m=1}^{2} g^{k m}\left(\frac{\partial g_{i m}}{\partial x_{j}}+\frac{\partial g_{j m}}{\partial x_{i}}+\frac{\partial g_{i m}}{\partial x_{m}}\right)
$$

See [9], $x_{1}=t$ and $x_{2}=\phi$, and $\left(g^{l m}\right)$ is the inverse matrix of $\left(g_{i j}\right)$. Then the scalar curvature of the surface $\chi(t, \phi)$ is

$$
\kappa=\sum_{i, j, l=1}^{2} g^{i j}\left[\frac{\partial \Gamma_{\mathrm{ij}}^{\mathrm{l}}}{\partial x_{l}}-\frac{\partial \Gamma_{\mathrm{il}}^{\mathrm{l}}}{\partial x_{j}}+\sum_{m=1}^{2}\left(\Gamma_{i j}^{l} \Gamma_{l m}^{m}-\Gamma_{i l}^{m} \Gamma_{j m}^{l}\right)\right]
$$

Notwithstanding the calculation of the scalar curvatureK can be accomplished, for example, by using the Mathematica programme, its explanation is some hulking. At the zero position $t=0$, the scalar curvature of $\chi(t, \phi)$ is given by:

$$
K=\frac{\mathrm{P}\left(\phi^{n} \cos m \phi, \phi^{n} \sin m \phi\right)}{Q\left(\phi^{n} \cos m \phi, \phi^{n} \sin m \phi\right)}=\frac{\sum_{m=0}^{2} \sum_{n=0}^{4}\left(A_{n, m} \phi^{n} \cos m \phi+B_{n, m} \phi^{n} \sin m \phi\right)}{\sum_{m=0}^{4} \sum_{n=0}^{7}\left(C_{n, m} \phi^{n} \cos m \phi+D_{n, m} \phi^{n} \sin m \phi\right)}
$$

The possibility the immutability of the scalar curvature $\mathrm{K}$ implies that Eq. (7) turns into:

$$
\mathrm{K} Q\left(\phi^{n} \cos m \phi, \phi^{n} \sin m \phi\right)-\mathrm{P}\left(\phi^{n} \cos m \phi, \phi^{n} \sin m \phi\right)=0
$$

Eq. (8) indicates that if we write it as a linear combination of the functions $\left\{\phi^{n} \cos m \phi, \phi^{n} \sin m \phi\right\} \quad$ namely, $\sum_{m=0}^{4} \sum_{n=0}^{7}\left(C_{n, m} \phi^{n} \cos m \phi+D_{n, m} \phi^{n} \sin m \phi\right)=0$, the comparable coefficients must vanish. Of here, we will be capable of describing all cycloid surfaces with fixed scalarcurvature obtained by the equiform motion of cycloid $m_{0}$. As we shall observe, it is not necessary to give the explanation of $\mathrm{K}$ but only the coefficients of higher order for the trigonometric functions. We know the cases $\mathrm{K}=0$.

\section{The Solution of Curvature Scalar in the Cycloid Curve}

In this part, we presume that $\mathrm{K}=0$ on the surface $\chi(t, \phi)$. From Eq. (7), we have: 


$$
\begin{aligned}
& \mathrm{P}\left(\phi^{n} \cos m \phi, \phi^{n} \sin m \phi\right)=\sum_{m=0}^{2} \sum_{n=0}^{4}\left(A_{n, m} \phi^{n} \cos m \phi+B_{n, m} \phi^{n} \sin m \phi\right)=0 \\
& Q\left(\phi^{n} \cos m \phi, \phi^{n} \sin m \phi\right)=\sum_{m=0}^{4} \sum_{n=0}^{7}\left(C_{n, m} \phi^{n} \cos m \phi+D_{n, m} \phi^{n} \sin m \phi\right) \neq 0
\end{aligned}
$$

$$
b_{j}^{\prime}=0 ; j=3,4,5
$$

cases, but we review this case $\mathrm{K}=0$ beneath this stipulation $s^{\prime} \neq 0$.

\subsection{Case $\boldsymbol{b}_{1}^{\prime} \boldsymbol{b}_{2}^{\prime} \neq \mathbf{0}$}

By solving the program Mathematica we get $\quad \alpha=b_{1}^{\prime 2}+b_{2}^{\prime 2}, \beta=s^{\prime} b_{1}^{\prime}-\omega_{1} b_{2}^{\prime}, \eta=s^{\prime} b_{2}^{\prime}+$ $\omega_{1} b_{1}^{\prime}, \gamma=s^{\prime 2}+\omega_{1}^{\prime 2}$. Then all coefficients $A_{n, m}=$ $b_{n, m}=0$ for all $0 \leq n \leq 2,0 \leq m \leq 4$ are trivial zero. Then the scalar curvature equal zero, from Eq. (7), we have:

$$
\begin{gathered}
b_{3}^{\prime 2}+b_{4}^{\prime 2}+b_{5}^{\prime 2}=0, \\
\omega_{2} b_{3}^{\prime}+\omega_{3} b_{4}^{\prime}+\omega_{4} b_{5}^{\prime}=0, \\
\omega_{5} b_{3}^{\prime}+\omega_{6} b_{4}^{\prime}+\omega_{7} b_{5}^{\prime}=0, \\
\omega_{2}^{\prime 2}+\omega_{3}^{\prime 2}+\omega_{4}^{\prime 2}=\omega_{5}^{\prime 2}+\omega_{6}^{\prime 2}+\omega_{7}^{\prime 2}=0 .
\end{gathered}
$$

Then we find;

$$
\omega_{i}=0 ; i=2,3,4, \ldots, 7
$$

$$
A(t)=\left(\begin{array}{ccccc}
\cos t & -\sin t & 0 & 0 & 0 \\
\sin t & \cos t & 0 & 0 & 0 \\
0 & 0 & \cos ^{2} t & -\sin t \cos t & -\sin t \\
0 & 0 & \sin t \cos t & \cos ^{2} t & \sin t \\
0 & 0 & \sin t \cos t & -\sin t & \cos ^{2} t
\end{array}\right)
$$

We presume $s^{\prime}(t)=e^{t}$ and $d(t)=(t, t, 0,0,0)^{\mathrm{T}}$, then $\omega_{1}=\omega_{8}=\omega_{9}=\omega_{10}=1$ and $\omega_{k}=0$ for $\sum_{k=2}^{7}$, $s^{\prime}=1, b_{1}^{\prime}=2, b_{2}^{\prime}=1 ; b_{3}^{\prime}=b_{4}^{\prime}=b_{5}^{\prime}=0$.

$$
X(t, \phi)=\left(\begin{array}{c}
2 t \\
t \\
0 \\
0 \\
0
\end{array}\right)+\left(\begin{array}{c}
1+t \\
-t \\
0 \\
0 \\
0
\end{array}\right)(\phi-\sin \phi)+\left(\begin{array}{c}
t \\
1+t \\
0 \\
0 \\
0
\end{array}\right)(1-\cos \phi)
$$

and

$$
Y(t, \phi)=\left(\begin{array}{c}
2 t \\
t \\
0
\end{array}\right)+\left(\begin{array}{c}
1+t \\
-t \\
0
\end{array}\right)(\phi-\sin \phi)+\left(\begin{array}{c}
t \\
1+t \\
0
\end{array}\right)(1-\cos \phi)
$$

\section{Two-Dimensional of a Cycloid Surfaces with $\mathrm{K} \neq \mathbf{0}$}

In this section, We assume that the scalar curvature
$\mathrm{K}$ of surfaces $\chi(t, \phi)$ given in

Eq. (2) is a non-zero steady . The identity Eq. (7) writes then as 


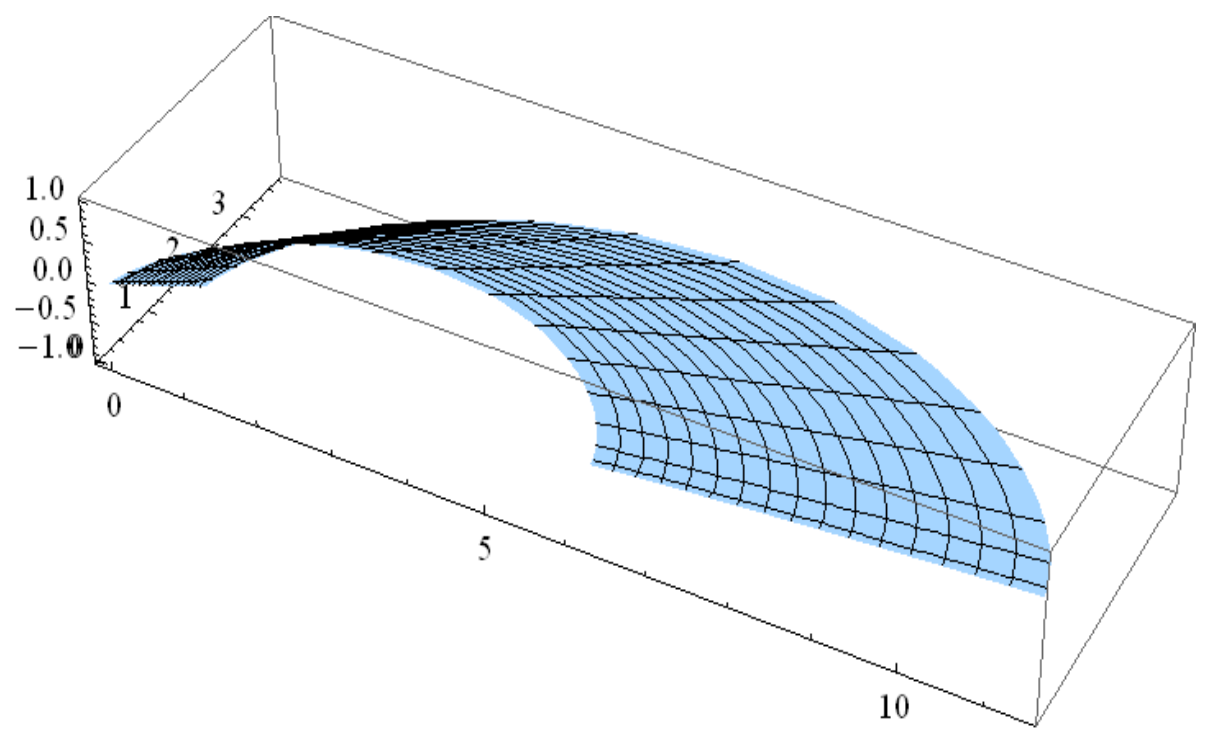

Fig. 1 we have a piece of a cycloid surface $X(t, \phi)$ with equation (2) that approximates.

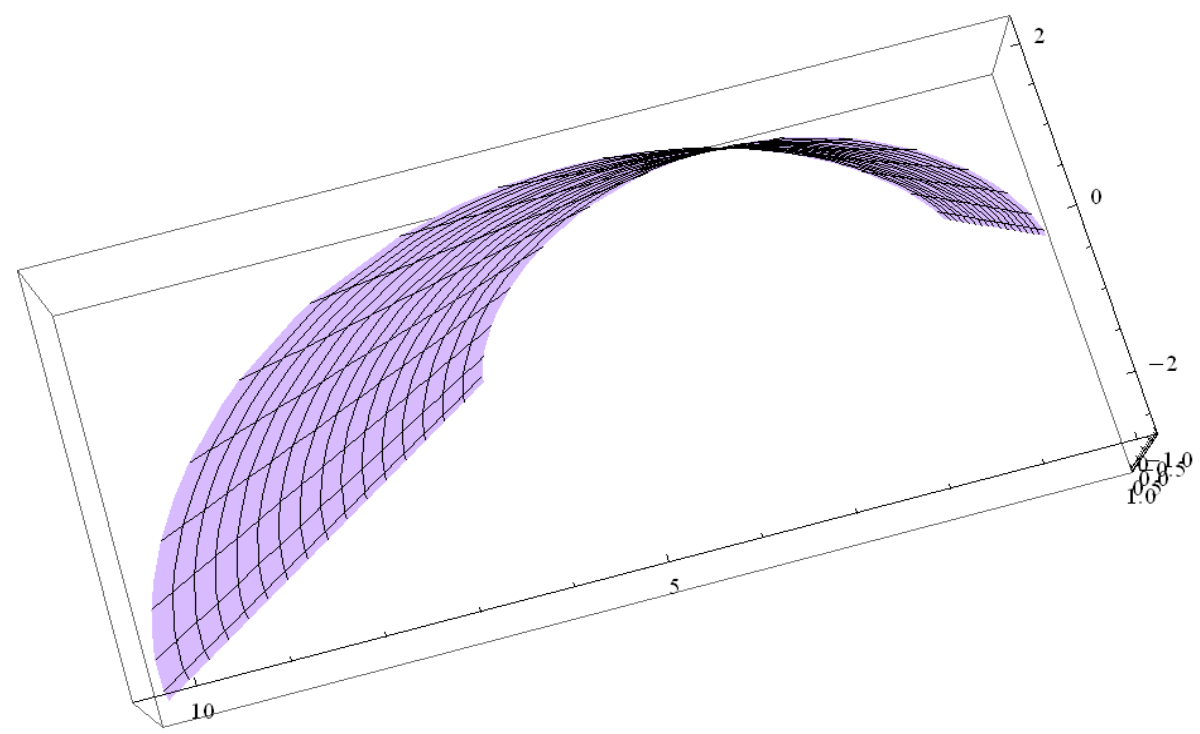

Fig. 2 we have a piece of a cycloid surface foliated by a cycloid curve in axonometric view $Y(t, \phi)$ with zero scalar curvature $\mathbf{K}=\mathbf{0}$.

$$
\sum_{m=0}^{4} \sum_{n=0}^{7}\left(C_{n, m} \phi^{n} \cos m \phi+D_{n, m} \phi^{n} \sin m \phi\right)=0
$$

We compute the coefficients $C_{n, m}, D_{n, m}$ in general case (For all $b_{1}, b_{2} \geq 0$ ) by using Mathematica programme and from equation (13) the coefficients $C_{n, m}, D_{n, m}$ must be vanish. Now we have $C_{2,5}=a^{8} \mu \mathrm{K}\left(s^{2}-\omega_{1}^{2}\right)=0$.

This coefficient equal zero if $\mu=0$ or $\omega_{1}^{2}=s^{\prime 2}$ both of them, we have to classify two cases: (a) If $\mu=0$. Then the coefficient $D_{4,4}=$ $a^{8} s^{\prime} \omega_{1} \mathrm{~K}\left(s^{\prime 2}-\omega_{1}^{2}\right)=0$. Implies that $\omega_{1}=0$ we find that the coefficient of $C_{4,4}=\frac{1}{4} a^{8} K s^{\prime 4}=0$ that leads to $\mathrm{K}=0$ or $s^{\prime}=0$ that is a ambivalence.

(b) From $C_{2,5}$ and $D_{4,4}$ we have $\omega_{1}^{2}=s^{2}$. Then the coefficient:

$$
C_{4,3}=2 a^{8} K s^{\prime 2}\left(-s^{2}+\gamma\right)=0
$$

we have $\gamma=s^{2}$, the coefficient of $D_{4,0}=$ $\frac{3}{4} a^{8} K s^{\prime 4}=0$. For $\mathrm{K} \neq 0$ and $s^{\prime} \neq 0$, it leads to 
inconsistency. As conclusion of the above consequence, we deduce the following theoremTheorem 5.1: There are not two-dimensional kinematic surfaces obtained by the equiform motion of a cycloid $m_{0}$ that given by Eq. (4) beneath Eq. (5) whose scalar curvature $K$ is a non-zero constant.

\section{A Domestic Isometry between 2-Dimensional Surfaces and 5-Dimensional Surfaces}

In this part, we will examine the presence of a local isometry among a two-dimensional surface in $\mathfrak{R}^{\mathbf{5}}$

$$
\bar{\chi}(t, \phi)=\left(\begin{array}{ccc}
1+\bar{s} t & t \bar{\omega}_{1} & t \bar{\omega}_{2} \\
-t \bar{\omega}_{1} & 1+\bar{s} t & t \bar{\omega}_{3} \\
-t \bar{\omega}_{2} & -t \bar{\omega}_{3} & 1+\bar{s} t
\end{array}\right)\left(\begin{array}{c}
a(\phi-\sin \phi) \\
a(1-\cos \phi) \\
0
\end{array}\right)+t\left(\begin{array}{l}
\bar{b}_{1} \\
\bar{b}_{2} \\
\bar{b}_{3}
\end{array}\right)
$$

The computation of the first fundamental form of $\bar{\chi}(t, \phi)$ indicates to:

$$
\begin{aligned}
& \bar{g}_{11}=\bar{\alpha}+2 a \bar{\beta}(\phi-\sin \phi)+a^{2} \bar{\gamma} \phi(\phi-\sin \phi)+a^{2} \bar{\mu}(\cos 2 \phi-1)+2 a(\bar{\eta}+a \bar{\delta})(1-\cos \phi) \\
& \bar{g}_{12}=a\left(\bar{b}_{1}^{\prime}+\bar{\beta} t+2 a \bar{\omega}_{1}+a \phi\left(\bar{s}^{\prime}+\bar{\gamma} t\right)\right)(1-\cos \phi)+a\left(\bar{b}_{1}^{\prime}+\bar{\eta} t-a \bar{\omega}_{1} \phi\right) \sin \phi+a^{2} \bar{\mu} t(2 \sin \phi-\sin 2 \phi)
\end{aligned}
$$

$$
\bar{g}_{22}=2 a^{2}\left(1+2 \bar{s}^{\prime} t+\bar{\gamma} t^{2}\right)(1-\cos \phi)+a^{2} \bar{\mu} t^{2}(1-\cos 2 \phi)
$$

and

$$
\begin{gathered}
\bar{\alpha}=\bar{b}_{1}^{\prime 2}+\bar{b}_{2}^{\prime 2}+\bar{b}_{3}^{\prime 2} \\
\bar{\beta}=\bar{s}^{\prime} \bar{b}_{1}^{\prime}-\bar{\omega}_{1} \bar{b}_{2}^{\prime}-\bar{\omega}_{2} \bar{b}_{3}^{\prime}, \\
\bar{\eta}=\bar{s}^{\prime} \bar{b}_{2}^{\prime}+\bar{\omega}_{1} \bar{b}_{1}^{\prime}-\bar{\omega}_{3} \bar{b}_{3}^{\prime}, \\
\bar{\gamma}=\bar{s}^{\prime 2}+\bar{\omega}_{1}^{\prime 2}+\bar{\omega}_{2}^{\prime 2}, \\
\bar{\delta}=\bar{s}^{2}+\bar{\omega}_{1}^{\prime 2}+\bar{\omega}_{3}^{\prime 2}, \\
\bar{\mu}=\frac{1}{2}\left(\bar{\omega}_{1}^{\prime 2}+\bar{\omega}_{3}^{\prime 2}\right) .
\end{gathered}
$$

In this case calculated $\mathfrak{R}^{\mathbf{5}}$, we have presumed that the original two axis of the cycloid are orthogonal. This means $\bar{\omega}_{2} \bar{\omega}_{3}=0$. On the other hand, the first fundamental form of $\chi(t, \phi)$ was studied in Eq. (4). From $\chi$ and $\bar{\chi}$, we have equations on the trigonometric functions $\sin (m \phi)$ and $\cos (m \phi)$.

The identities $g_{i j}=\overline{g_{\imath \jmath}}$ imply $s^{\prime}=\bar{s}^{\prime 2}$, $\omega_{1}=\bar{\omega}_{1}, \quad b_{1}^{\prime}=\bar{b}_{1}^{\prime}, \quad b_{2}^{\prime}=\bar{b}_{2}^{\prime}$ and $\alpha=\bar{\alpha}, \beta=\bar{\beta}$, $\gamma=\bar{\gamma}, \delta=\bar{\delta}, \eta=\bar{\eta}, \mu=\bar{\mu}, \lambda=\bar{\lambda}, \bar{\omega}=0$.

Thus:

$$
\begin{gathered}
b_{4}^{\prime 2}+b_{5}^{\prime 2}=0, \\
\bar{\omega}_{2}^{\prime 2}=\omega_{2}^{\prime 2}+\omega_{3}^{\prime 2}+\omega_{4}^{\prime 2}, \\
\bar{\omega}_{3}^{\prime 2}=\omega_{5}^{\prime 2}+\omega_{6}^{\prime 2}+\omega_{7}^{\prime 2},
\end{gathered}
$$

$$
\begin{aligned}
& \bar{\omega}_{2} \bar{b}_{3}^{\prime}=\omega_{2} b_{3}^{\prime}+\omega_{3} b_{4}^{\prime}+\omega_{4} b_{5}^{\prime}, \\
& \bar{\omega}_{3} \bar{b}_{3}^{\prime}=\omega_{5} b_{3}^{\prime}+\omega_{6} b_{4}^{\prime}+\omega_{7} b_{5}^{\prime} .
\end{aligned}
$$

Theorem 6.1: Examine a kinematic surface in $\mathfrak{R}^{\mathbf{5}}$ given by the parametrization $\chi(t, \phi)$ in Eq. (3) beneath condition (4) and with constant scalar curvature. Let $\bar{\chi}(t, \phi)$ be a kinematic surface in $\mathfrak{R}^{3}$ specified by Eq. (14). If the following equations:

$$
s^{\prime}=\bar{s}^{\prime 2}, \omega_{1}=\bar{\omega}_{1}, b_{1}^{\prime}=\bar{b}_{1}^{\prime}, b_{2}^{\prime}=\bar{b}_{2}^{\prime}, b_{4}^{\prime 2}+b_{5}^{\prime 2}=0 .
$$

Then both surfaces $\chi(t, \phi)$ and $\$ \backslash$ bar $\bar{\chi}(t, \phi)$ are locally isometric. The Gaussian curvature of the surface $\bar{\chi}(t, \phi)$ in Euclidean space $\mathfrak{R}^{3}$ must vanish.

\section{References}

[1] Do Carmo, M. 1976. "Differential Geometry of Curves and Surfaces.” Prentice-Hall Inc. Englewood Cliffs, New Jersey.

[2] Solouma, E. M. 2015. "Three-Dimensional Surfaces Foliated by an Equiform Motion of Pseudohyperbolic Surfaces in $\mathfrak{R}^{7}$." Journal of JP Geometry and Topology 17 (2): 109-26.

[3] Solouma, E. M. 2007. "Three-Dimensional Surfaces Foliated by Two-Dimensional Spheres." J. of Egyptian Mathematical Society 1: 101-10. 
[4] Wageeda, M. M. 2015. "Some Properties of the Two-Dimensional Kinematic Surfaces Obtained by an Equiform Motion of a Sinusoidal Curve." IJARSET 2 (7): 736-43.

[5] Bottema, O. and Roth, B. 1990. "Theoretical Kinematic." Dover Publications Inc., New York.

[6] Pottmann, H. and Wallner, J. 2001. "Computational Line Geometry.” Springer-Verlag, Heidelberg, Berlin.

[7] Solouma, E. M. 2012. "A Local Study of Scalar Curvature of Two-Dimensional Surfaces Obtained by the Motion of Circle." J. of Applied Math. and Computation
219:3385-94.

[8] Kreyszig, E. 1975. "Introduction to Differential Geometry and Riemannian Geometry." University of Toronto Press, Canada.

[9] O’Neill, B. 1983. "Semi-Riemannian Geometry with Application to Relativity." Academic Press.

[10] Solouma, E. M., Wageeda, M. M., Gouda, Y. Gh. and Bary, M. 2015. "Studying Scalar Curvature of Two-Dimensional Kinematic Surfaces Obtained by Using Similarity Kinematic of a Deltoid." Applied Mathematics 6: 1353-61. 